\title{
ABO phenotypes and inflammation-related predictors of lung cancer mortality: the Copenhagen Male Study - a 16-year follow-up
}

\author{
P. Suadicani*, H.O. Hein*,* and F. Gyntelberg*
}

ABSTRACT: Inflammation and genetic susceptibility influence the risk of lung cancer. Previous studies suggest that the inflammatory response may depend upon ABO phenotype.

The hypothesis that the association with lung cancer mortality risk of lifestyle and occupational factors previously linked to inflammation would depend upon $A B O$ phenotype was tested in a long-term follow-up of 3,346 male subjects aged 53-74 yrs.

During a 16-yr period, $170(5.1 \%)$ of the male subjects died due to lung cancer; $84(5.9 \%)$ of phenotype $0,70(4.9 \%)$ of phenotype $A$ and $16(3.2 \%)$ of phenotype B/AB. In addition to cumulative tobacco consumption, high salt intake long-term occupational dust exposure, high fat intake and consumption of alcohol were significantly predictive of lung cancer mortality for phenotype $\mathrm{O}$ subjects. After multivariable adjustment, the hazard ratios associated with the first three of these factors were 2.31, 2.08 and 1.67, respectively. Compared with abstainers, the hazard ratios for males drinking 1-10 wine drinks week $^{-1}$ and males drinking $>10$ wine drinks week $^{-1}$ were 1.65 and 2.02, respectively. Among phenotype A subjects, only cumulative tobacco consumption was associated with lung cancer mortality risk.

The predictive role of inflammation-related risk factors for lung cancer mortality was significantly stronger among males of phenotype $\mathrm{O}$ than $\mathrm{A}$.

KEYWORDS: ABO phenotypes, alcohol use, epidemiology, lung cancer mortality, salt intake

I nflammation and the nature of the inflammatory response seem to be important determinants in the pathogenesis of lung cancer [1-4]. How is it, though, that recognition of this fact has led to the analysis of potential differences in risk predictors among male subjects with different $\mathrm{ABO}$ phenotypes?

In the Copenhagen Male Study (CMS), a relatively strong interplay was found between occupational airborne pollutants, ABO phenotype and risk of ischaemic heart disease (IHD), with long-term exposure being associated with a significantly increased risk among males of phenotype $\mathrm{O}$, but not among males with other ABO phenotypes [5]. SJÖGREN [6] suggested that occupational exposure to particulate air pollution may increase the risk of cardiovascular disease since it results in release of inflammatory mediators, including interleukin (IL)-6, which, in turn, stimulates the production of fibrinogen in the liver. Furthermore, it has been shown that individuals of blood type $\mathrm{O}$ show an increased inflammatory response when exposed to Helicobacter pylori, with a significantly increased release of IL-6 [7]. The CMS supported the concept that, compared with other ABO phenotypes, males of phenotype $\mathrm{O}$ may exhibit a stronger systemic inflammatory response following occupational exposure to particulate air pollution [5].

In another recent article, YUDKIN et al. [8] suggested that similar inflammatory mediators, specifically IL-6, may be a link connecting obesity and coronary heart disease. This suggestion was extended in the CMS, and it was hypothesised that the strength of association between longterm occupational exposure to air pollutants and risk of obesity might also be modified by $\mathrm{ABO}$ phenotype, with those of phenotype $\mathrm{O}$ being particularly susceptible. This hypothesis was supported inasmuch as a lifetime history of long-term occupational exposure to numerous potentially respirable air pollutants was associated with a higher prevalence of obesity in this group; no such association was observed among males with other $\mathrm{ABO}$ phenotypes [9].

The increasing recognition of inflammation as a contributing factor to the development of cancer,
AFFILIATIONS

*The Copenhagen Male Study, Epidemiological Research Unit, Clinic of Environmental and Occupational Medicine, Bispebjerg University Hospital, and

${ }^{\#}$ Research Centre for Prevention and Health, Glostrup University Hospital, Copenhagen, Denmark.

CORRESPONDENCE

P. Suadicani

Copenhagen Male Study Epidemiological Research Unit Clinic of Environmental and Occupational Medicine Bispebjerg University Hospital Bispebjerg Bakke 23 DK-2400 Copenhagen NV Denmark

Fax: 4535316070

E-mail: PS11@bbh.hosp.dk

Received:

May 102006

Accepted after revision:

March 142007

SUPPORT STATEMENT

This study was supported by the King Christian X Foundation, Danish Medical Research Council, Danish Heart Foundation and Else and Mogens Wedell-Wedellsborg Foundation (all Copenhagen, Denmark).

STATEMENT OF INTEREST None declared. 
along with the above observations [1-9], motivated the hypothesis that the role of inflammation-related risk factors for lung cancer may depend upon ABO phenotype.

\section{MATERIAL AND METHODS}

\section{Patient characteristics}

The CMS was set up, in 1970/1971, as a prospective cardiovascular cohort study of 5,249 Caucasian male subjects with a mean age of 48 yrs (range $40-59$ yrs). The examination comprised a short interview based on a previously completed questionnaire, from which information was obtained concerning lifestyle, including smoking habits [10, 11]. Based on information about education and job profile, the males were subdivided into five social classes, as previously described [12].

In 1985/1986 a new baseline was established, giving more comprehensive information about the study participants. This baseline was used for the present study. All males from the 1970/1971 study were traced by means of the Danish Central Population Register (Copenhagen, Denmark). Between June 1985 and June 1986, all survivors (except for 34 emigrants) from the original cohort were invited to take part in the present study. In total, 3,387 (75\%) male subjects agreed and gave informed consent; their mean age was 63 yrs (range 53-74 yrs). The 1985/1986 study took place at The Glostrup Population Studies (Glostrup Hospital, University of Copenhagen, Copenhagen, Denmark). Each subject was interviewed about a previously completed questionnaire and subjected to a clinical examination; a venous blood sample was taken for determination of $\mathrm{ABO}$ phenotype. With respect to $\mathrm{ABO}$ phenotype and lung cancer mortality diagnoses, useful information was available for 3,346 of the males. For no other single variable included did the proportion of missing values exceed $3 \%$.

\section{ABO phenotype}

$\mathrm{ABO}$ determination, including reverse typing, was performed using conventional in vitro haemagglutination techniques. As previously described, the distribution of ABO phenotypes did not deviate from previous Danish studies carried out on blood bank and medico-legal specimens [13].

\section{Tobacco smoking habits}

The male subjects classified themselves as never-smokers, previous smokers or current smokers. Current tobacco consumption was calculated from information about the number of cigarettes, cheroots or cigars, or the weight of pipe tobacco smoked daily. One cigarette was taken to be equivalent to $1 \mathrm{~g}$ tobacco, one cheroot to $3 \mathrm{~g}$ tobacco and one cigar as $4 \mathrm{~g}$ tobacco. As previously estimated by means of serum cotinine levels, the validity of tobacco reporting in the CMS was high [14]. Cumulative tobacco consumption (in pack-years) was calculated on the basis of information about mean total daily use of tobacco in grams multiplied by the time as a smoker in years divided by 20. In the multivariable analyses, cumulative tobacco consumption was included as a predictor using quintile categories; the cut-off points were 3.9, 19.0, 32.0 and 45.0 pack-yrs, respectively.

\section{Dietary factors}

Salt and fat intake were crudely measured based on the two following simple questions. Do you use more salt in your food than others? Do you try to avoid fatty food?

\section{Alcohol consumption}

Total weekly alcohol consumption was calculated from questionnaire items about mean alcohol consumption on weekdays and at weekends. Intakes of beer, wine and spirits were reported separately. One drink corresponded to 10-12 g ethanol.

\section{Occupational exposures}

Information about potentially relevant physical and chemical respirable occupational exposures was obtained from the questionnaire. Long-term exposure was defined as frequent occupational exposure for $\geqslant 5$ yrs. The question was phrased as follows. "Have you, at your present or previous workplaces, often, i.e. several times/week or more, been exposed to dust, asbestos, etc.? If yes, for how many years?" Exposures for which $\geqslant 3 \%$ of the cohort had reported long-term exposure were considered relevant for scrutiny in stratified analyses; 10 different exposures were included in the study.

\section{End-points}

Notifications of death and causes of death within the period 1985/1986 to the end of 2001 were obtained from the Danish Register of Causes of Death. The International Classification of Diseases (ICD) lung cancer mortality diagnoses used were codes162 of ICD-8, and, from 1994, C34 of ICD-10. Information about the histological type of lung cancer was available for 128 of the 170 lung cancer deaths. No differences were found with respect to histology between $\mathrm{ABO}$ phenotypes; the size of histological subgroups did not permit further stratified analyses.

\section{Statistical analysis}

Basic statistical analyses, including an unpaired t-test, Chisquared analysis, Mann-Whitney rank-sum test and regression analyses, were performed. The log rank test was used to test equality of survival distributions in life tables. Relative risks were estimated by $\exp (\beta)$, where $\beta$ is the hazard coefficient for the variable of interest in a Cox's proportional-hazards regression model, using the maximum likelihood ratio method and a backward stepwise elimination procedure [15]. Assumptions regarding the use of Cox's proportional hazards were met by inspection of the $\log$ minus $\log$ function at the covariate mean. In order to test the relative importance of lung cancer mortality predictors between the two major phenotypes, groups $\mathrm{O}$ and $\mathrm{A}$, a number of separate analyses were performed, including multiplicative interaction terms between phenotype $\mathrm{O}$ or $\mathrm{A}$ and the factors in question. Relevant interaction terms were included in a final analysis comprising males of both phenotype $\mathrm{O}$ and phenotype A. The interaction term consisted of the result of a multiplication of the genetic risk factor (coded 1 for phenotype $\mathrm{O}$ and 0 for phenotype $\mathrm{A}$ ) and the risk factor (coded 1 for present and 0 for absent for salt intake, fat intake and dust exposure, and, with respect to wine intake, coded 2 for intake of $>10$ wine drinks $\cdot$ week $^{-1}, 1$ for 1 10 wine drinks. week ${ }^{-1}$ and 0 for no intake). The rationale behind the analysis of interaction has been described by 
KLeINBAum et al. [16]. A two-sided probability value of $\mathrm{p} \leqslant 0.05$ was a priori taken as significant.

\section{Ethics}

Each participant was informed that all personal data were confidential, and gave written consent to participation. The present study was approved by the Committee for Ethics in Medical Research in the County of Copenhagen.

\section{RESULTS}

Overall, in the population eligible for study, 170 (5.1\%) males died due to lung cancer during the period 1985/1986 to the end of 2001, 5.9\% among males of phenotype O, $4.9 \%$ of phenotype $\mathrm{A}$ and $3.2 \%$ of either phenotype $\mathrm{B}$ or $\mathrm{AB}, \mathrm{a}$ significant difference between the three groups on Cox's proportional-hazards analysis, adjusting for age $(\mathrm{p}<0.05)$. Referencing phenotype A subjects, the lung cancer mortality hazard ratio for phenotype $\mathrm{O}$ subjects was $1.18 \quad(95 \%$ confidence interval (CI) 0.86-3.55), and, referencing phenotype $\mathrm{B} / \mathrm{AB}$ subjects, it was 2.05 (1.18-3.55).

\section{Baseline characteristics among different ABO phenotype subjects}

Table 1 shows the univariate characteristics, stratified according to $\mathrm{ABO}$ phenotype, of subjects who died due to lung cancer and of others, i.e. those who either died due to causes other than lung cancer or were survivors at the end of the 16-yr follow-up period. Since there were only a small number of cases among males of $\mathrm{B}$ and $\mathrm{AB}$ phenotype, these phenotypes were pooled, and interpretation of the results of these analyses was accordingly more conservative.
Lung cancer covariates were remarkably different among males of different ABO phenotype. Strong associations with lung cancer were found for both phenotype $\mathrm{O}$ and A subjects with respect to smoking characteristics; this association was stronger for phenotype O subjects. Only one subject (1.2\%) out of 84 cases had never smoked among phenotype $\mathrm{O}$ males, whereas seven $(10 \%)$ were never-smokers among phenotype $\mathrm{A}$ males, a significant difference $(\mathrm{p}=0.02$ (Chi-squared test)).

Phenotype $\mathrm{O}$ males who died due to lung cancer exhibited higher total alcohol consumption than others; this was not the case among phenotype $\mathrm{A}$ or $\mathrm{B} / \mathrm{AB}$ males. On univariate analysis of type of alcoholic beverage consumed among males with phenotype $\mathrm{O}$, the strongest discrimination between lung cancer cases and others was found for intake of strong alcoholic beverages, i.e. spirits.

Among phenotype $\mathrm{O}$ males who died due to lung cancer, $28.6 \%$ had given an affirmative answer to the question regarding whether they had a higher salt intake than others. Among phenotype $\mathrm{O}$ males who did not die due to lung cancer, only $11.9 \%$ gave a positive answer to this question $(\mathrm{p}<0.001)$. Among phenotype O subjects, $66.7 \%$ of those who died due to lung cancer reported not avoiding fat as compared to $48.6 \%$ among noncases, a highly significant difference.

Among phenotype A subjects, no difference was found between lung cancer cases and others with respect to reported salt intake. The proportion $(65.7 \%)$ of lung cancer cases who reported not avoiding fat intake was comparable to that observed for phenotype $\mathrm{O}$ subjects, whereas a larger proportion

TABLE 1 Lifestyle characteristics of males who died due to lung cancer and other causes during the period 1985/1986-2001 according to $\mathrm{ABO}$ phenotype

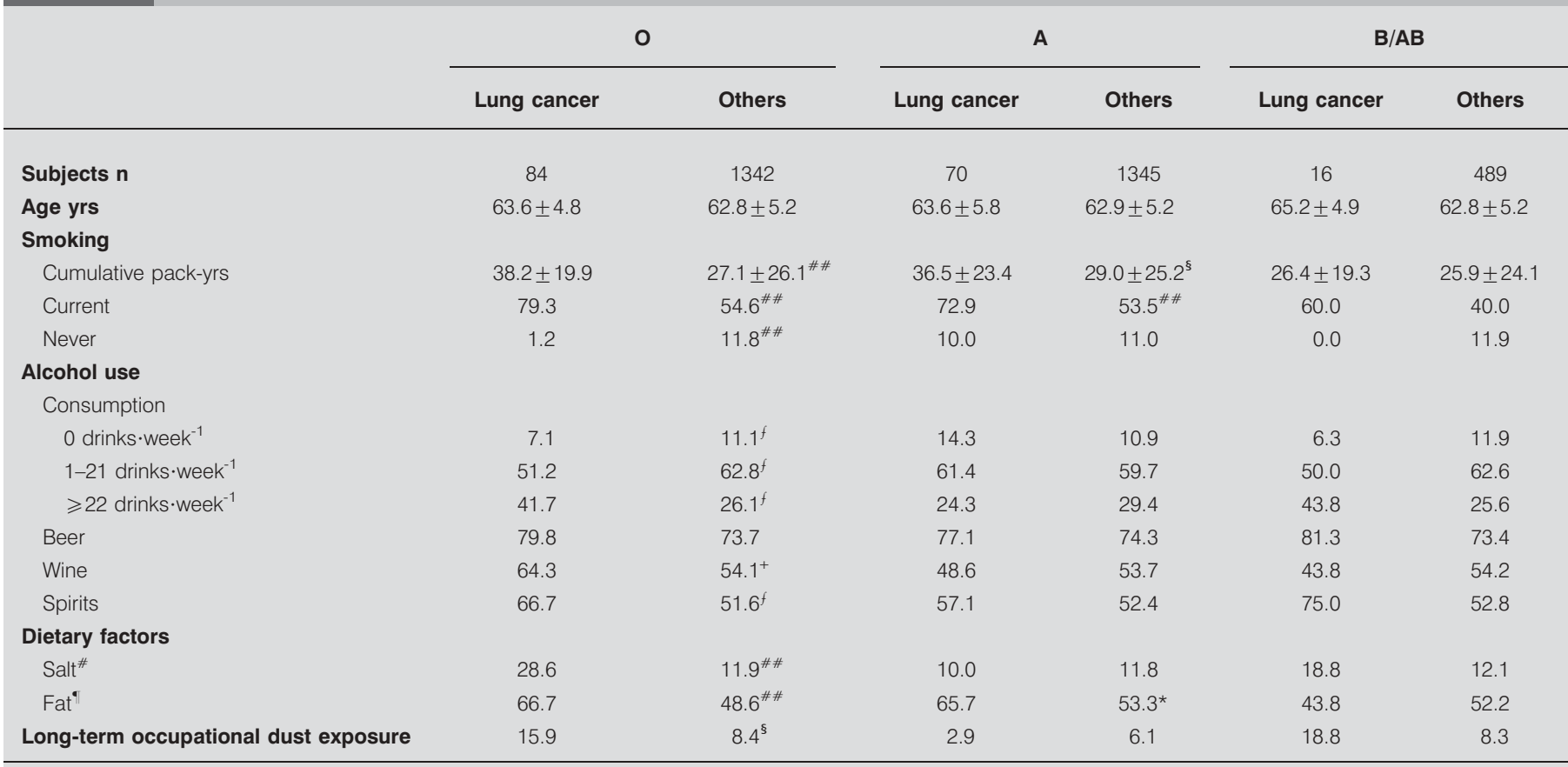

Data are presented as mean \pm SD or percentages, unless otherwise stated. ${ }^{\#}:$ uses more than others; ${ }^{\circ}$ : does not avoid fatty foods. ${ }^{+}: p=0.07 ;{ }^{s}: p \leqslant 0.05 ;{ }^{f}: p \leqslant 0.01$, this is an overall value, lung cancer cases were significantly unevenly distributed in the three groups; $\# \#$ : $p \leqslant 0.001$ (unpaired t-test or Chi-squared test as appropriate). 
(53.3\%) of noncases reported not avoiding fat compared with phenotype $\mathrm{O}$ subjects.

The association with lung cancer mortality of long-term occupational exposure to 10 more-or-less specific potentially respirable occupational factors stratified according to $\mathrm{ABO}$ phenotype was analysed (data not shown). The percentages of males exposed to the various factors for $\geqslant 5$ yrs were as follows: 1) dust (from cement, building demolition, mineral fibres, etc.) $7.6 \%$; 2) asbestos $3.4 \%$; 3) soldering fumes $8.1 \%$; 4 ) welding, casting or blowpipe fumes $8.2 \%$; 5) organic solvents $9.0 \%$; 6) fumes from paint, lacquer or varnish $5.4 \%$; 7) toxic components $5.3 \%$; 8) breath irritants $7.5 \%$; 9) stench or strongsmelling products $8.9 \%$; and 10) irritants (other than breath irritants or contagious components) or chemicals $4.1 \%$. In separate Cox's proportional hazards analyses with adjustment for age, long-term occupational dust exposure was revealed to be significant among phenotype $\mathrm{O}$ subjects, and was associated with an almost two-fold higher risk of lung cancer mortality (hazard ratio 1.97; 95\% CI 1.09-3.57). Thus dust exposure was regarded as relevant for further study. As shown in table 1, among phenotype $\mathrm{O}$ subjects, $15.9 \%$ of lung cancer cases had received long-term exposure to occupational dust compared with $8.4 \%$ among noncancer cases $(\mathrm{p}<0.05)$.

\section{Occupational and nonoccupational predictors among different $A B O$ phenotype subjects}

Table 2 shows the relative predictive role of the potentially relevant risk factors identified in table 1. Among males of phenotype $\mathrm{O}$, after adjustment, the strongest association with lung cancer was found for cumulative cigarette consumption (in pack-years). In addition, self-assessed high salt consumption, long-term occupational dust exposure, high fat intake and wine intake were significantly associated with lung cancer mortality among males of phenotype $\mathrm{O}$.

With respect to phenotype A subjects, only cumulative cigarette consumption and age were significant predictors. On analysis of phenotype B and AB subjects, only increasing age was significantly associated with risk of lung cancer mortality, and a tendency was found with respect to history of long-term exposure to dust.

\section{Predictors among phenotype $\mathrm{O}$ and $\mathrm{A}$ subjects, including interaction terms}

Table 3 shows the result of Cox's proportional hazards regression analysis including only the two most prevalent phenotypes, $\mathrm{O}$ and $\mathrm{A}$. As shown in table 2, significant predictors for phenotype $\mathrm{O}$ males, but not phenotype A males, were high salt intake, long-term occupational dust exposure, fat intake and wine intake, and a tendency was found towards a stronger association of cumulative cigarette consumption with lung cancer mortality among males of phenotype $\mathrm{O}$ than among males of phenotype A. In order to test whether these predictors of lung cancer were significant between the two phenotypes, separate analyses were performed using one multiplicative interaction term in each analysis. In order to form the interaction terms, $\mathrm{O} / \mathrm{A}$ phenotype was multiplied by each of the following: high salt intake; dust exposure; wine intake; fat intake; and cumulative cigarette consumption. Four of these multiplicative interaction terms were identified as potentially relevant for the final analysis presented in table 3 . After multivariable adjustment, the strongest predictive factor was cumulative cigarette consumption quintile. Three interaction terms remained in the final model, those involving high salt intake, wine intake and dust exposure. The main effects of wine intake, spirits intake, beer intake, long-term dust

\section{TABLE 2}

Cox's proportional regression models of lifestyle and occupational characteristics significantly associated with lung cancer mortality after multivariable adjustment according to ABO phenotype ${ }^{\#}$

\begin{tabular}{|c|c|c|c|}
\hline & 0 & A & $B / A B$ \\
\hline \multicolumn{4}{|l|}{ Cumulative cigarette consumption } \\
\hline $\mathrm{Q1}^{\#}$ & $1 ; 289$ & $1 ; 256$ & \\
\hline Q2 & $1.41(0.45-4.45) ; 295$ & $0.66(0.24-1.87) ; 270$ & \\
\hline Q3 & $3.85(1.42-10.47) ; 272$ & $1.33(0.57-3.11) ; 300$ & \\
\hline Q4 & $5.01(1.90-13.19) ; 285$ & $2.38(1.08-5.22) ; 294$ & \\
\hline Q5 & $5.02(1.90-13.22) ; 273$ & $2.23(1.01-4.94) ; 283$ & \\
\hline Salt versus not & $2.31(1.40-3.83)$ & & \\
\hline Age risk per 1-yr increment & $1.06(1.02-1.11)$ & $1.05(1.01-1.10)$ & $1.14(1.03-1.26)$ \\
\hline Long-term occupational dust exposure versus not ${ }^{+}$ & $2.08(1.15-3.78)$ & & $2.87(0.80-10.30)$ \\
\hline Fat versus others ${ }^{f}$ & $1.67(1.03-2.72)$ & & \\
\hline \multicolumn{4}{|l|}{ Wine intake } \\
\hline 0 drinks $\cdot$ week $^{-1 \#}$ & 1 & & \\
\hline $1-10$ drinks $\cdot$ week $^{-1}$ & $1.65(0.99-2.73)$ & & \\
\hline$>10$ drinks $\cdot$ week $^{-1}$ & $2.02(1.11-3.68)$ & & \\
\hline
\end{tabular}

Data are presented as hazard ratio (95\% confidence interval), followed by number of subjects for quintile (Q) data (or hazard ratio and number of subjects alone for the reference category (Q1)). The independent covariates in all analyses were age, high salt intake, fat intake (does not avoid), beer intake, wine intake, spirits intake, cumulative cigarette consumption (quintiles) and long-term dust exposure. The factors were ranked according to the strength of the association with the outcome after adjustment; factors with a p-value of $>0.10$ after adjustment were excluded. ${ }^{*}$ : reference category; ${ }^{*}$ : uses more than others; ${ }^{+}$: from cement, building demolition, mineral fibres, etc. ${ }^{f}$ : does not avoid fatty foods. 


\begin{tabular}{|c|c|c|}
\hline \multirow[t]{2}{*}{ TABLE 3} & \multicolumn{2}{|c|}{$\begin{array}{l}\text { Risk factors for lung cancer mortality during the } \\
\text { period 1985/1986-2001 among phenotype O } \\
\text { and A males }\end{array}$} \\
\hline & & Hazard ratio $(95 \% \mathrm{Cl})$ \\
\hline \multicolumn{3}{|c|}{ Cumulative cigarette consumption } \\
\hline $\mathrm{Q} 1^{\#}$ & & $1 ; 545$ \\
\hline Q2 & & 0.93 (0.44-1.98); 565 \\
\hline Q3 & & $2.18(1.16-4.13) ; 572$ \\
\hline Q4 & & 3.33 (1.82-6.08); 579 \\
\hline Q5 & & $3.23(1.76-5.93) ; 556$ \\
\hline Age risk per & -yr increment & $1.06(1.02-1.09)$ \\
\hline Salt $\times 0 / A$ & henotype $^{+}$ & $2.34(1.43-3.85)$ \\
\hline Fat versus o & ers $^{f}$ & $1.58(1.12-2.24)$ \\
\hline \multicolumn{3}{|c|}{ Wine intake $\times$ O/A phenotype ${ }^{+}$} \\
\hline 0 drinks $\cdot w e$ & $\mathrm{~K}^{-1} / \mathrm{O}$ and all $\mathrm{A}$ males ${ }^{\#}$ & 1 \\
\hline $1-10$ drinks & veek $^{-1} / O$ versus reference category & $1.54(0.76-3.15)$ \\
\hline$>10$ drinks & reek $^{-1} / O$ versus reference category & $4.38(1.60-11.96)$ \\
\hline $\begin{array}{l}\text { LT occupatic } \\
\text { phenotype }\end{array}$ & lal dust ${ }^{\S}$ exposure $\times$ O/A & $2.07(1.14-3.76)$ \\
\hline O versus $A$ & lenotype & $0.59(0.35-1.00)$ \\
\hline
\end{tabular}

Quintile (Q) data are followed by the number of subjects, with hazard ratio and number of subjects alone being presented for the reference category (Q1). The same predictors as in table 2, as well as relevant interaction terms, were included in this model. The Cox's proportional hazard ratios are presented according to the strength of the association with lung cancer mortality after multivariable adjustment. Factors with a p-value of $>0.10$ after adjustment (the main effects of wine intake, spirits intake, beer intake, long-term occupational dust exposure and high salt intake, and the interaction of fat intake and O/A phenotype) were excluded from the final model. Cl: confidence interval; LT: long-term. ${ }^{*}$ : reference category; ${ }^{\bullet}$ : uses more than others; ${ }^{+}$: interaction term; ${ }^{f}$ : does not avoid fatty

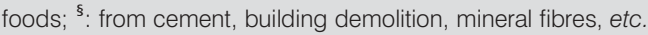

exposure and high salt intake, and the interaction of fat intake and $\mathrm{O} / \mathrm{A}$ phenotype, were excluded. A similar analysis including cumulative cigarette consumption as a continuous variable instead of in discreet quintiles gave a result in complete accordance with the one presented.

\section{Illustrating the relative importance over time}

Figure 1 illustrates the association with lung cancer mortality of

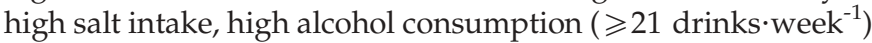
and long-term occupational dust exposure on separate analysis of males of phenotype $\mathrm{O}$. The presence of one or more of these factors was associated with an excess risk of lung cancer mortality over the entire period of follow-up among males of phenotype $\mathrm{O}$, whereas no association was seen among males of phenotype A (data not shown). Among males of phenotype $\mathrm{O}$, the association was even more pronounced if males who had never smoked were excluded from the analysis. Compared to phenotype $\mathrm{O}$ males without any of these risk factors, the hazard ratio in a Cox's analysis with adjustment for age and cumulative cigarette consumption was 2.46 (1.54-3.93) for those with at least one of the risk factors. With $40.5 \%$ of phenotype $\mathrm{O}$ males having at least one of these risk factors, the population-attributable risk was calculated to be $37 \%$ (95\% CI $18-54 \%$ ); in a corresponding analysis including only phenotype

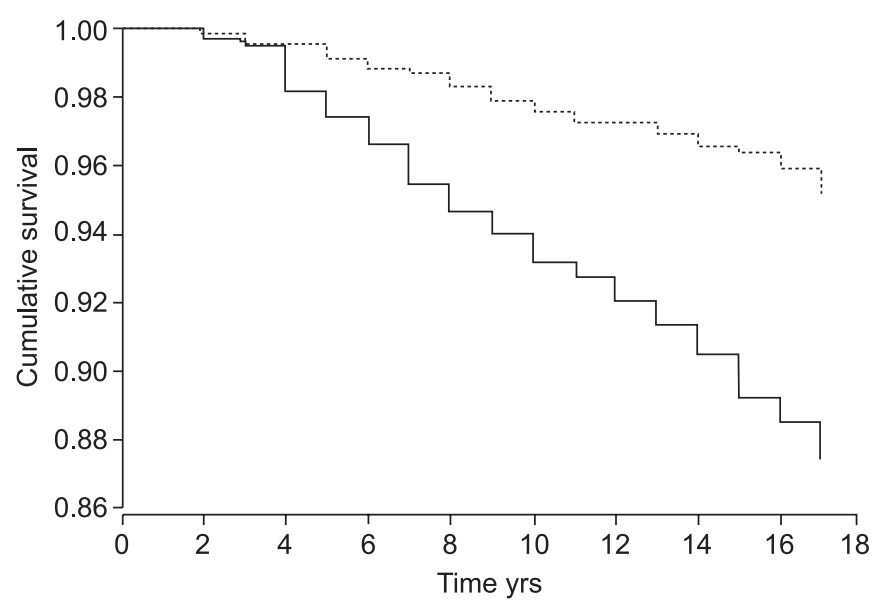

FIGURE 1. Deaths due to lung cancer over a 16-yr period among phenotype O males with any $(-)$ or none $(\cdots \cdots)$ of the following risk factors: high salt intake, high alcohol consumption or dust exposure. $p<0.001$.

O males who were smokers at baseline $(1985 / 1986)$, the population-attributable risk was 32\% (95\% CI 10-52\%).

\section{DISCUSSION}

The predictive role of risk factors for lung cancer mortality was strongly influenced by or dependent upon ABO phenotype. Among males of phenotype $\mathrm{O}$, in addition to smoking history, three nontobacco lifestyle factors and one occupational factor were independent predictors of death due to lung cancer. High salt consumption, high alcohol intake and long-term occupational dust exposure (to essentially inorganic dust) were significant predictors of lung cancer mortality among phenotype $\mathrm{O}$ subjects alone, as indicated by the significant interactions and illustrated in figure 1.

Among males of phenotype A, smoking (cumulative cigarette consumption) was the only significant lifestyle risk factor in the multivariable model. Although the number of lung cancer cases among males of phenotype B/AB was small, a few observations should be commented upon. As a group, these males showed a significantly lower risk of lung cancer mortality compared with those of phenotype O. Furthermore, a tendency was also found towards occupational dust exposure being a risk factor for this group. Since there were a small number of end-points among males of phenotype B or $\mathrm{AB}$, testing of the relative importance of risk factors for lung cancer mortality in the present study focused on differences between the major phenotypes, $\mathrm{O}$ and $\mathrm{A}$.

\section{Tobacco smoking}

Some conspicuous findings concerning the relative significance of smoking among phenotype $\mathrm{O}$ and $\mathrm{A}$ males were seen. Of those who died from lung cancer among males of phenotype A, $10 \%$ had never smoked compared to only $1 \%$ among males of phenotype O. Low levels of serum cotinine at baseline, and no history of smoking in 1970/1971 within this group, supported their claim to be never-smokers. In addition, the association of serum cotinine levels at baseline differed between the two phenotypes, with a stronger association with lung cancer mortality among phenotype $\mathrm{O}$ subjects (data not shown). 


\section{High salt intake}

There has been little focus on the potentially adverse effects on lung cancer risk as a result of a high intake of salt. This is illustrated by the fact that a PubMed search using various combinations of terms relating to table salt intake and lung cancer returned no articles. However, some indirect evidence linking high salt intake with risk of lung cancer exists. A casecontrol study in Japan found an increased risk of lung cancer among people with high consumption of miso soup, one of the leading sources of sodium chloride for Japanese people [17, 18]. Another case-control study of 320 lung cancer cases and 320 controls concluded that "Also, salted meat consumption and beer intake were associated with an increased risk of lung cancer" [19].

\section{High alcohol intake}

Alcohol consumption was proposed as a risk factor for lung cancer in 1984 [20]. Although most studies since then have found an excess risk of lung cancer with a high level of alcohol intake, the issue has remained controversial due to the difficulties in controlling sufficiently for tobacco use. In the present study, a strong positive association between level of alcohol consumption and lung cancer was found for phenotype $\mathrm{O}$ subjects, with $42 \%$ of lung cancer cases consuming $\geqslant 21$ drinks week $^{-1}$ as compared with $26 \%$ of noncases; among phenotype A males, the association was not significant and the corresponding proportions were marginally different, 24 and $29 \%$, respectively. Taking into account information on tobacco habits did not change these relative differences in the predictive role of alcohol. In a previous Danish study, an overall higher risk of lung cancer was found among those drinking spirits, and a protection against development of lung cancer was observed among wine drinkers [21].

\section{Long-term dust exposure}

Long-term occupational dust exposure (from cement, building demolition, mineral fibres, etc.) was significantly associated with risk of lung cancer mortality among phenotype $\mathrm{O}$ but not phenotype A males. Asbestos is a well-known risk factor for lung cancer, and silica dust exposure has also been associated with lung cancer in susceptible individuals [22]. The results of the present study identify phenotype $\mathrm{O}$ subjects as a particularly susceptible group.

\section{Fat intake}

With respect to fat intake, the present findings are in accordance with the majority of the existing literature, showing a high fat intake to be associated with increased risk of lung cancer [23]. However, the relative importance of fat intake as a predictor only differed marginally between phenotypes $\mathrm{O}$ and A (table 1). The validity of this crude information about fat intake was supported by results from an analysis of the association between obesity and fat intake, showing a strong inverse relationship among males genetically at high risk of obesity, and a much weaker association for males genetically at low risk (unpublished data from the CMS).

\section{Biological plausibility}

All of the above factors have been associated with inflammation. Salt intake has previously been associated with respiratory symptoms, with personal table salt use being strongly related to cough and phlegm [24]. Recently, a double-blind randomised experimental study including asthma patients showed an enhanced inflammatory response in subjects with the highest dietary salt load [25]. In the CMS, further analyses were performed on the association between self-assessed high salt intake and chronic bronchitis, defined according to the definition of the UK General Medical Council [26], and between chronic bronchitis and lung cancer mortality (data not shown). A high salt intake was associated with an approximately two-fold higher prevalence of chronic bronchitis among smokers; this association was independent of ABO phenotype (data not shown). Chronic bronchitis was independently, i.e. adjusted for smoking history and other risk factors included in the present study, associated with a significant two-fold higher risk of lung cancer mortality compared with males without bronchitis, an observation in complete accordance with a recent analysis of the Copenhagen City Heart Study [27]. In addition, occupational dust exposure is an established risk factor for chronic bronchitis [22, 26]. Use of diuretics is associated with increased urinary sodium excretion. In support of a possibly causal relationship of high salt intake with lung cancer mortality among phenotype $\mathrm{O}$ subjects, even the predictive role of diuretics use for lung cancer was dependent upon ABO phenotype. Among males of phenotype $\mathrm{O}$, the cumulative incidence among 131 males using diuretics was 2.3 versus $6.4 \%$ among males not using diuretics $(\mathrm{p}=0.035)$; among males of phenotype A, 128 took diuretics, and the corresponding figures were 5.5 and $4.9 \%$, respectively $(p=0.78)$. This additional analysis indirectly supports the methodological validity of the very simple assessment of salt intake used in the present study.

High alcohol consumption has been shown to be associated with chronic bronchitis. In the CMS, the existence of a J- or Ushaped relationship of alcohol consumption to prevalence of chronic bronchitis has previously been shown [26]. In a study including blood donors, a U-shaped relationship was observed between sensitive markers of inflammation (C-reactive protein, serum amyloid A and IL-6) and alcohol consumption [28].

In addition, the intake of fatty acids has been associated with the levels of several biomarkers of inflammation, including IL6 and C-reactive protein, as shown in a recent study [29].

It has previously been suggested that increased levels of IL-6 following long-term occupational exposure to air pollutants might be the mechanism explaining the increased risk of IHD, as well as obesity, found among males of phenotype $\mathrm{O}$ in the CMS $[5,9]$. Interestingly, CARUSO et al. [30] recently hypothesised that low IL-6 levels may control inflammatory regulation and cancer development better. Accordingly, although still speculative, the results of the present study are in agreement with the previous findings of the present authors, namely that males of phenotype $\mathrm{O}$ exhibit a different physiological response compared with others when exposed to inflammation-inducing agents.

\section{Methodological considerations}

Information regarding smoking, use of alcohol and dust exposure in the CMS has previously been found to be of high validity $[14,26,31]$. A few of the exposure measures are crude, such as the dietary markers of salt intake and fat intake. This 
coarse and qualitative assessment would, however, tend not to explain but rather diminish the association with outcome. In addition, with respect to the potential risk factors examined, marginal differences were observed between $\mathrm{ABO}$ phenotypes in the overall distribution of these factors.

Do males reporting a high salt intake really consume more salt than others, or do their answers to the simple question on salt intake reflect a merely subjective perception? No objective measures of urinary salt excretion were available, so these questions cannot be answered in the present study. The conclusion to be drawn from the observations on dietary salt, phenotype $\mathrm{O}$ and risk of lung cancer is that phenotype $\mathrm{O}$ males with a history of smoking giving an affirmative answer to a simple question on having a high salt intake exhibit an increased risk of lung cancer. It seems unlikely that underlying confounding factors explain the very consistent findings regarding the above association. The present study at least raises the hypothesis that a high salt intake may be an important, potentially modifiable, risk factor for lung cancer among males of phenotype $\mathrm{O}$ with a history of smoking.

\section{Conclusion}

The predictive role of inflammation-related risk factors for lung cancer mortality was stronger among males of phenotype O than phenotype A.

\section{REFERENCES}

1 Ardies CM. Inflammation as cause for scar cancers of the lung. Integr Cancer Ther 2003; 2: 238-246.

2 Philip M, Rowley DA, Schreiber H. Inflammation as a tumor promoter in cancer induction. Semin Cancer Biol 2004; 14: 433-439.

3 Knaapen AM, Borm PJ, Albrecht C, Schins RP. Inhaled particles and lung cancer. Part A: mechanisms. Int J Cancer 2004; 109: 799-809.

4 Emmendoerffer A, Hecht M, Boeker T, Mueller M, Heinrich U. Role of inflammation in chemical-induced lung cancer. Toxicol Lett 2000; 112-113: 185-191.

5 Suadicani P, Hein HO, Gyntelberg F. Airborne occupational exposure, $\mathrm{ABO}$ phenotype and risk of ischaemic heart disease in the Copenhagen Male Study. J Cardiovasc Risk 2002; 9: 191-198.

6 Sjögren B. Occupational exposure to dust, inflammation and ischaemic heart disease. Occup Environ Med 1997; 54: 466-469.

7 Alkout AM, Blackwell CC, Weir DM. Increased inflammatory responses of persons of blood groups $\mathrm{O}$ to Helicobacter pylori. J Infect Dis 2000; 181: 1364-1369.

8 Yudkin JS, Kumari M, Humphries SE, Mohamed-Ali V. Inflammation, obesity, stress and coronary heart disease: is interleukin-6 the link? Atherosclerosis 2000; 148: 209-214.

9 Suadicani P, Hein HO, Gyntelberg F. Airborne occupational exposure, $\mathrm{ABO}$ phenotype and risk of obesity. Int J Obes 2005; 29: 689-696.

10 Gyntelberg F. Physical fitness and coronary heart disease in male residents in Copenhagen aged 40-59. Dan Med Bull 1973; 20: 1-4.
11 Gyntelberg F. One- and two-years incidence of myocardial infarction in Copenhagen males aged 40-59. Dan Med Bull 1975; 22: 81-84.

12 Hein HO, Suadicani P, Gyntelberg F. Lung cancer risk and social class. The Copenhagen Male Study - 17-year follow up. Dan Med Bull 1992; 39: 173-176.

13 Mourant AE, Kopec AC, Damaniewska-Sobcnoak K. The Distribution of the Human Blood Groups and Other Polymorphisms. London, Oxford University Press, 1976.

14 Suadicani P, Hein HO, Gyntelberg F. Serum validated tobacco use and social inequalities in risk of ischaemic heart disease. Int J Epidemiol 1994; 23: 293-300.

15 Cox DR. Regression models and life-tables. J $R$ Stat Soc 1972; 34: 187-220.

16 Kleinbaum DG, Kupper LL, Morgenstern H, Interaction, effect modification, and synergism. In: Kleinbaum DG, Kupper LL, Morgenstern H. Epidemiological Research. Principles and Quantitive Methods. New York, John Wiley and Sons Inc, 1982; pp. 403-418.

17 Watanabe T, Miyasaka M, Koizumi A, Ikeda M. Regional differences in sodium chloride content in home-made and store-bought preparations of miso paste. Tohoku J Exp Med 1982; 137: 305-313.

18 Wakai K, Ohno Y, Genka K, et al. Risk modification in lung cancer by dietary intake of preserved foods and soyfoods: findings from a case-control study in Okinawa, Japan. Lung Cancer 1999; 25: 147-159.

19 De Stefani E, Deneo-Pellegrini H, Carzoglio JC, Roco A, Mendilaharsu M. Dietary nitrosodimethylamine and the risk of lung cancer: a case-control study from Uruguay. Cancer Epidemiol Biomarkers Prev 1996; 5: 679-682.

20 Bandera EV, Freudenheim JL, Vena JE. Alcohol consumption and lung cancer: a review of the epidemiologic evidence. Cancer Epidemiol Biomarkers Prev 2001; 10: 813-821.

21 Prescott E, Gronbaek M, Becker U, Sorensen TI. Alcohol intake and the risk of lung cancer: influence of type of alcoholic beverage. Am J Epidemiol 1999; 149: 463-470.

22 Hnizdo E, Vallyathan V. Chronic obstructive pulmonary disease due to occupational exposure to silica dust: a review of epidemiological and pathological evidence. Occup Environ Med 2003; 60: 237-243.

23 Fabricius PG, Lange P. Kost og lungekræft. [Diet and lung cancer.]. Ugeskr Laeger 2003; 165: 3234-3237.

24 Pistelli R, Forastiere F, Corbo GM, et al. Respiratory symptoms and bronchial responsiveness are related to dietary salt intake and urinary potassium excretion in male children. Eur Respir J 1993; 6: 517-522.

25 Mickleborough TD, Lindley MR, Ray S. Dietary salt, airway inflammation, and diffusion capacity in exerciseinduced asthma. Med Sci Sports Exerc 2005; 37: 904-914.

26 Suadicani P, Hein HO, Meyer HW, Gyntelberg F. Exposure to cold and draught, alcohol consumption, and the NSphenotype are associated with chronic bronchitis: an epidemiological investigation of 3387 men aged 5375 years: the Copenhagen Male Study. Occup Environ Med 2001; 58: 160-164.

27 Lange P, Parner J, Prescott E, Vestbo J. Chronic bronchitis in an elderly population. Age Ageing 2003; 32: 636-642.

28 Hoffmeister A, Imhof A, Rothenbacher D, Khuseyinova N, Brenner H, Koenig W. Moderater Alkoholkonsum und Plasmakonzentration sensitiver Entzündungsmarker - 
Hinweise auf einen atheroprotektiven Zusammenhang. [Moderate alcohol consumption and plasma concentration of sensitive markers of inflammation. Comment on an atheroprotective relationship.]. Dtsch Med Wochenschr 2003; 128: 2237-2241.

29 Lopez-Garcia E, Schulze MB, Meigs JB, et al. Consumption of trans fatty acids is related to plasma biomarkers of inflammation and endothelial dysfunction. I Nutr 2005; 135: 562-566.

30 Caruso C, Lio D, Cavallone L, Franceschi C. Aging, longevity, inflammation, and cancer. Ann N Y Acad Sci 2004; 1028: 1-13.

31 Hein HO, Suadicani P, Sørensen H, Gyntelberg F. Alcohol consumption, Lewis phenotypes, and risk of ischaemic heart disease. Lancet 1993; 341: 392-396. 